\title{
ECC
}

Eurasian Chemical Communications

\section{Programming of Fe-catalyzed cascade Knoevenagel-Michael- cyclocondensation reaction: create out pseudo acridine derivatives under solvent free conditions}

\author{
Ardeshir Khazaeia,*, Ahmad Reza Moosavi-Zareb,*, Hadis Afshar- \\ Hezarkhania, Vahid Khakyzadehc \\ a Faculty of Chemistry, Bu-Ali Sina University, Hamedan, 6517838683, Iran \\ ${ }^{b}$ Department of Chemistry, Sayyed Jamaleddin Asadabadi University, Asadabad, 6541861841, Iran \\ cDepartment of Chemistry, K. N. Toosi University of Technology, P.O. BOX 15875 - 4416, Tehran, Iran
}

Received: 13 October 2019, Accepted: 02 November 2019, Published: 23 November 2019

\begin{abstract}
Nano-ferrous ferric oxide (nano- $\mathrm{Fe}_{3} \mathrm{O}_{4}$ ) was successfully applied as an efficient and heterogeneous catalyst for the synthesis of 1,8-dioxo-decahydroacridines by the condensation reaction of dimedone with aromatic aldehydes and ammonium acetate or aniline derivatives under mild and solvent-free conditions. Also, optimization of the reaction condition was investigated using the response surface method (Central Composite Design (CCD) \} compared to empirical research.
\end{abstract}

Keywords: $\mathrm{Nano}_{-} \mathrm{Fe}_{3} \mathrm{O}_{4}$; cascade reaction; 1,8-dioxo-decahydroacridine; heterogeneous catalyst.

\section{Introduction}

Multi-component reactions (MCRs) have significant role in combinatorial chemistry due to the ability to afford expected products with high efficiency and atomic economy by the reaction of three or more compounds together in a one step. Also, MCRs increase simplicity and synthetic efficiency on the conventional organic transformations [15].

Organic transformations catalyzed by transition-metal are often followed the principles of "Green Chemistry" due to minimization of waste and energy, cleaner auxiliaries, catalysts and reagents [6]. Magnetite nanoparticles (MNPs) are very attractive catalysts due to their magnetic properties and high specific surface area $[7,8]$. They can be easily collected with a magnet to reuse it to proceed another reactions. Recently, the chemists focused their attantion on catalytic aspects of nano- $\mathrm{Fe}_{3} \mathrm{O}_{4}$ to modify methods of catalytic activity [9$14]$.

1, 8-Dioxo-decahydroacridines are polyfunctionalized 1, 4-dihydropyridine derivatives which have received less attention than other 1, 4-DHP derivatives [15]. Several methods have been reported for the synthesis of 1,8-dioxodecahydroacridine derivatives [16-31]. However, some of them suffer from the

*Corresponding author: Ardeshir Khazaei, Ahmad Reza Moosavi-Zare

Tel: +98 (65) 17838683, Fax: +98 (65) 17838683

E-mail: Khzaei_1326@yahoo.com,moosavizare@yahoo.com

Eurasian Chem. Commun., (2020) 27-34 
drawbacks such as the use of toxic metals, the use of volatile organic solvents, high cost and low yields. Solvent-free reactions have been illustrated to be as an efficient technique for different organic transformations instead of using harmful organic solvents [32-37].

Herein, we have introduced the solvent-free synthesis of 1, 8-dioxo-

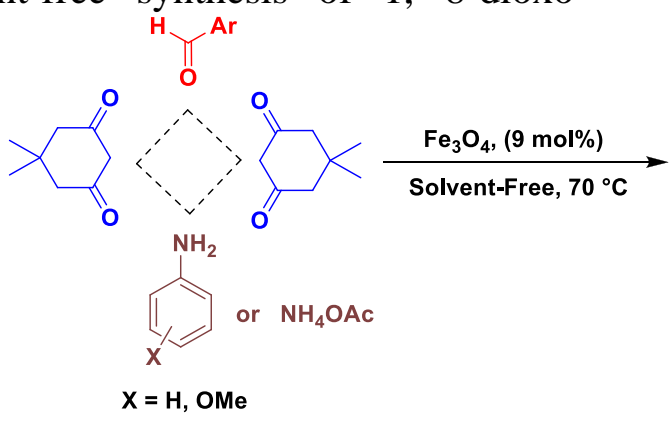

decahydroacridine derivatives in the presence of Nano- $\mathrm{Fe}_{3} \mathrm{O}_{4}$ as an efficient, heterogeneous and reusable catalyst with high yields and short reaction times (Scheme 1). Optimization of the reaction condition was investigated using the response surface method \{Central Composite Design (CCD) \}.

Scheme 1. The synthesis of 1,8-dioxo-decahydroacridines

\section{Experimental}

All chemicals were purchased from Merck or Fluka Chemical Companies. All known compounds were identified by comparison of their melting points and spectral data with those reported in the literature. Progress of the reactions was monitored by thin layer chromatography (TLC) using silica gel SIL G/UV 254 plates. The melting points were recorded on a Büchi B-545 apparatus in open capillary tubes. The ${ }^{1} \mathrm{H}$ NMR (400 MHz) and ${ }^{13} \mathrm{C}$ NMR (100) were run on a Bruker Avance DPX, FTNMR spectrometers, $\delta$ in ppm.

General procedure for the synthesis of 1, 8-dioxo-decahydroacridines using nano- $\mathrm{Fe}_{3} \mathrm{O}_{4}$ :

A mixture of dimedone ( $0.28 \mathrm{~g}, 2 \mathrm{mmol})$, aldehyde $(1 \mathrm{mmol})$, ammonium acetate (or aniline derivatives) ( $1 \mathrm{mmol}, 0.077$ $\mathrm{g})$, and nano- $\mathrm{Fe}_{3} \mathrm{O}_{4}(9 \mathrm{~mol} \%)$ in a $25 \mathrm{~mL}$ round-bottomed flask connected to a reflux condenser was stirred magnetically. After completion of the reaction as monitored by TLC, the mixture was cooled to room temperature and warm EtOH $(30 \mathrm{~mL})$ was added, stirred and refluxed for 4 minutes. The catalyst was collected by a magnet and separated from the solution of the product and remaining starting materials and reused for the next run. The crude product was purified by recrystallization from ethanol (95\%).

9-(2, 3-Dichlorophenyl)-3, 3, 6, 6tetramethyl-3, 4, 6, 7, 9, 10hexahydroacridine-1, $8(2 \mathrm{H}, 5 \mathrm{H})$-dione (4)

${ }^{1} \mathrm{H}$ NMR $\left(400 \mathrm{MHz}, \mathrm{CDCl}_{3}\right) \delta=1.10(\mathrm{~s}$, $\left.6 \mathrm{H}, 2 \mathrm{CH}_{3}\right), 1.23\left(\mathrm{~s}, 6 \mathrm{H}, 2 \mathrm{CH}_{3}\right), 2.19(\mathrm{~s}$, $\left.2 \mathrm{H}, \mathrm{CH}_{2}\right), 2.28(\mathrm{~s}, 2 \mathrm{H}), 2.34$ (d, J=6.80 $\mathrm{Hz}, 1 \mathrm{H}), 2.39$ (s, 1H), 2.43 (s, 1H), 2.48 (s, 1H ), 5.49 (s, 1H ), 7.03 (s, 2H, ArH ), 7.28 (s, 1H, ArH ), 11.16 (s , 1H, NH). ${ }^{13} \mathrm{C}$ NMR (100 MHz, $\left.\mathrm{CDCl}_{3}\right) \delta=27.5$, 29.6, 31.4, 32.4, 46.4, 47.0, 115.5, 126.7, $135.5,189.3,190.5$.

3, 3, 6, 6-Tetramethyl-9-(2nitrophenyl)-3, 4, 6, 7, 9, 10hexahydroacridine-1, $8(2 \mathrm{H}, 5 \mathrm{H})$-dione (6)

${ }^{1} \mathrm{H}$ NMR: $\left(400 \mathrm{MHz}, \mathrm{CDCl}_{3}\right) \delta=1.03(\mathrm{~s}$, $\left.6 \mathrm{H}, 2 \mathrm{CH}_{3}\right), 1.16\left(\mathrm{~s}, 6 \mathrm{H}, 2 \mathrm{CH}_{3}\right), 2.21(\mathrm{~s}$, $\left.2 \mathrm{H}, \mathrm{CH}_{2}\right), 2.25(\mathrm{~s}, 1 \mathrm{H}, \mathrm{CH}), 2.33$ (d, 
$\left.J=16.00 \mathrm{~Hz}, 2 \mathrm{H}, \mathrm{CH}_{2}\right), 2.43(\mathrm{~s}, 1 \mathrm{H}, \mathrm{CH})$, $2.48(\mathrm{~d}, J=10.00 \mathrm{~Hz}, 1 \mathrm{H}, \mathrm{CH}), 2.53$ (s, $1 \mathrm{H}, \mathrm{CH}), 6.05(\mathrm{~s}, 1 \mathrm{H}, \mathrm{CH}), 7.27(\mathrm{t}$, $J=4.40 \mathrm{~Hz}, 1 \mathrm{H}, \mathrm{ArH}), 7.34(\mathrm{t}, J=7.60 \mathrm{~Hz}$, $1 \mathrm{H}, \mathrm{ArH}), 7.490$ (t, J=8.00 Hz, 1H, ArH), $7.61(\mathrm{~d}, J=8.40 \mathrm{~Hz}, 1 \mathrm{H}, \mathrm{ArH}), 11.60$ (s, $1 \mathrm{H}, \mathrm{NH}) ;{ }^{13} \mathrm{C}$ NMR $\left(100 \mathrm{MHz}, \mathrm{CDCl}_{3}\right) \delta$ 28.1, 28.5, 30.0, 31.9, 46.2, 46.8, 114.6, 124.3, 127.1, 129.5, 131.3, 132.1, 149.7, 189.1, 190.1.

\section{3, 3, 6, 6-Tetramethyl-9-(naphthalen-} 2-yl)-3, 4, 6, 7, 9, 10hexahydroacridine- $1,8(2 \mathrm{H}, 5 \mathrm{H})$-dione (8)

${ }^{1} \mathrm{H}$ NMR $\left(400 \mathrm{MHz}, \mathrm{CDCl}_{3}\right) \delta=1.15(\mathrm{~s}$, $\left.6 \mathrm{H}, 2 \mathrm{CH}_{3}\right), 1.32\left(\mathrm{~s}, 6 \mathrm{H}, 2 \mathrm{CH}_{3}\right), 2.34$ $(\mathrm{s}, 1 \mathrm{H}), 2.40(\mathrm{~d}, J=12.00 \mathrm{~Hz}, 1 \mathrm{H}), 2.45(\mathrm{~s}$, $1 \mathrm{H}), 2.51(\mathrm{~s}, 1 \mathrm{H}), 2.55(\mathrm{~s}, 1 \mathrm{H}), 5.70(\mathrm{~s}$, $1 \mathrm{H}, \mathrm{CH}), 7.26(\mathrm{t}, J=8.80 \mathrm{~Hz}, 1 \mathrm{H}), 7.41-$ $7.46(\mathrm{~m}, 1 \mathrm{H}), 7.53(\mathrm{~s}, 1 \mathrm{H}), 7.71-7.80(\mathrm{~m}$, 4H) , $11.96(\mathrm{~s}, 1 \mathrm{H}, \mathrm{NH}) ;{ }^{13} \mathrm{C}$ NMR $\left(100 \mathrm{MHz}, \mathrm{CDCl}_{3}\right) \delta=27.4,29.6,31.5$, 33.0, 46.4, 47.1, 115.6, 125.3, 125.9,
$127.4, \quad 127.8, \quad 127.8, \quad 131.8, \quad 133.2$, 135.5,189.5, 190.5.

9, 9'-(1, 4-Phenylene)bis (3, 3, 6, 6tetramethyl-3, 4, 6, 7, 9, 10hexahydroacridine- $1, \quad 8(2 \mathrm{H}, \quad 5 \mathrm{H})$ dione) (9)

${ }^{1} \mathrm{H}$ NMR (400 MHz, DMSO- $\left.d_{6}\right) \delta=0.85$ $\left(\mathrm{s}, 6 \mathrm{H}, 2 \mathrm{CH}_{3}\right), 0.94\left(\mathrm{~s}, 6 \mathrm{H}, 2 \mathrm{CH}_{3}\right), 0.93$ $\left(\mathrm{s}, 6 \mathrm{H}, 2 \mathrm{CH}_{3}\right), 1.03\left(\mathrm{~s}, 6 \mathrm{H}, 2 \mathrm{CH}_{3}\right), 2.03$ $(\mathrm{m}, 2 \mathrm{H}), 2.14(\mathrm{~s}, 2 \mathrm{H}), 2.24(\mathrm{~d}, J=9.60$ $\mathrm{Hz}, 2 \mathrm{H}), 2.30$ (s, 2H ), 2.37 (d, $J=15.60$ $\mathrm{Hz}, 2 \mathrm{H}), 2.51$ (d, $J=11.60 \mathrm{~Hz}, 2 \mathrm{H}), 2.67$ (d, $J=12.80 \mathrm{~Hz}, 2 \mathrm{H}), 3.16$ (s , 2H), 4.59 $(\mathrm{s}, 2 \mathrm{H}, 2 \mathrm{CH}), 5.86(\mathrm{~s}, 1 \mathrm{H}, \mathrm{NH}), 6.98(\mathrm{~s}$, 1H, ArH), 7.06-7.19 (m, 2H, ArH), 7.38 (d, $J=7.60 \mathrm{~Hz}, 1 \mathrm{H}, \mathrm{ArH}), 10.95$ (s, $1 \mathrm{H}$, ArH).

\section{Results and discussion}

Nano- $\mathrm{Fe}_{3} \mathrm{O}_{4}$ was prepared according to the previous literature [13]. Transmission electron microscopy (TEM) of nano- $\mathrm{Fe}_{3} \mathrm{O}_{4}$ was studied to confirm that the obtained particles were prepared in nano size (Figure 1).

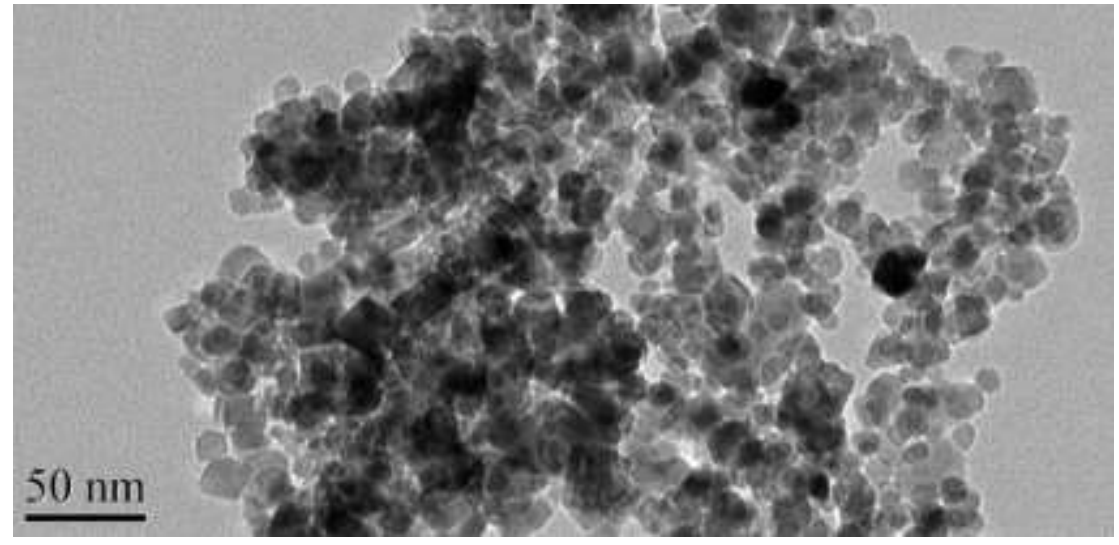

Figure 1. Transmission electron microscopy (TEM) of the nano- $\mathrm{Fe}_{3} \mathrm{O}_{4}$ =

Initial studies with programming of the reaction, to find the optimal condition for the synthesis of 1,8-dioxodecahydroacridine derivatives by the reaction of dimedone $(2 \mathrm{mmol})$ with 2 nitrobenzaldehyde $(1 \mathrm{mmol})$ and ammonium acetate $(1.2 \mathrm{mmol})$, as a model reaction, were started. The response surface method \{Central Composite Design (CCD) $\}$ with three replicates at central point was selected and a second order model was developed. We found that temperature and amount of catalyst are two key factors in terms of selectivity, time and yield. Levels of the variables (investigation area) for temperature (A) and amount of catalyst (B) were 25 to $100{ }^{\circ} \mathrm{C}$ and 1 to $10 \mathrm{~mol} \%$ respectively. Also, dependent variables including 
yield and time of the reaction were suitable for fitting to a quadratic polynomial model (Table 1).

Table 1. Levels of the experimental variables and the corresponding response values of the CCD

\begin{tabular}{ccccc}
\hline \multirow{2}{*}{ Run } & \multicolumn{2}{c}{ Independent variables } & \multicolumn{2}{c}{ Dependent variables } \\
\cline { 2 - 5 } & $\mathbf{A}\left({ }^{\mathbf{0}} \mathbf{C}\right)$ & $\mathbf{B}(\mathbf{m o l} \%)$ & Time (min) & Yield $(\boldsymbol{\%})$ \\
\hline $\mathbf{1}$ & 25 & 5.5 & 15 & 85 \\
$\mathbf{2}$ & 25 & 1 & 30 & 50 \\
$\mathbf{3}$ & 62.5 & 5.5 & 4 & 95 \\
$\mathbf{4}$ & 62.5 & 5.5 & 4 & 95 \\
$\mathbf{5}$ & 100 & 10 & 3 & 95 \\
$\mathbf{6}$ & 62.5 & 1 & 20 & 50 \\
$\mathbf{7}$ & 100 & 5.5 & 3 & 95 \\
$\mathbf{8}$ & 62.5 & 5.5 & 4 & 95 \\
$\mathbf{9}$ & 25 & 10 & 15 & 85 \\
$\mathbf{1 0}$ & 62.5 & 10 & 4 & 95 \\
$\mathbf{1 1}$ & 100 & 1 & 15 & 50 \\
\hline
\end{tabular}

Based on significant levels and actual values, two polynomial response surface models were obtained as follows;

Time: $3.087 \mathrm{~A} 2+0.362 \mathrm{~B} 2-0.559 \mathrm{~A}-$ $5.580 \mathrm{~B}+46.884$ (Equation 1)
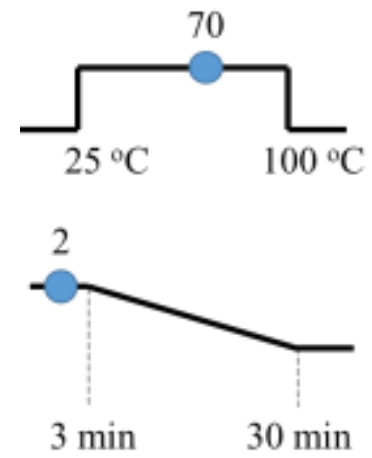

Yield: -2.619 A2 - $1.0461 \mathrm{~B} 2+0.148$ $\mathrm{AB}+0.334 \mathrm{~A}+15.211 \mathrm{~B}+$ 26.668(Equation 2)

After optimization studies and programing, optimal point was found at A $\left(70^{\circ} \mathrm{C}\right)$ and B (9.1) (Figure 2).
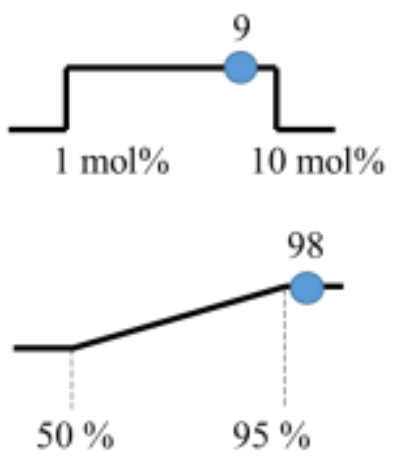

Figure 2. Introduced optimal point of variables

In Figure 3, two and threedimensional profiles of yield from the model are illustrated. It can be seen from time and yield equations that the amount of catalyst has the main linear effect on the temperature of the reaction and there is a significant interaction between the amount of catalyst and temperature. 

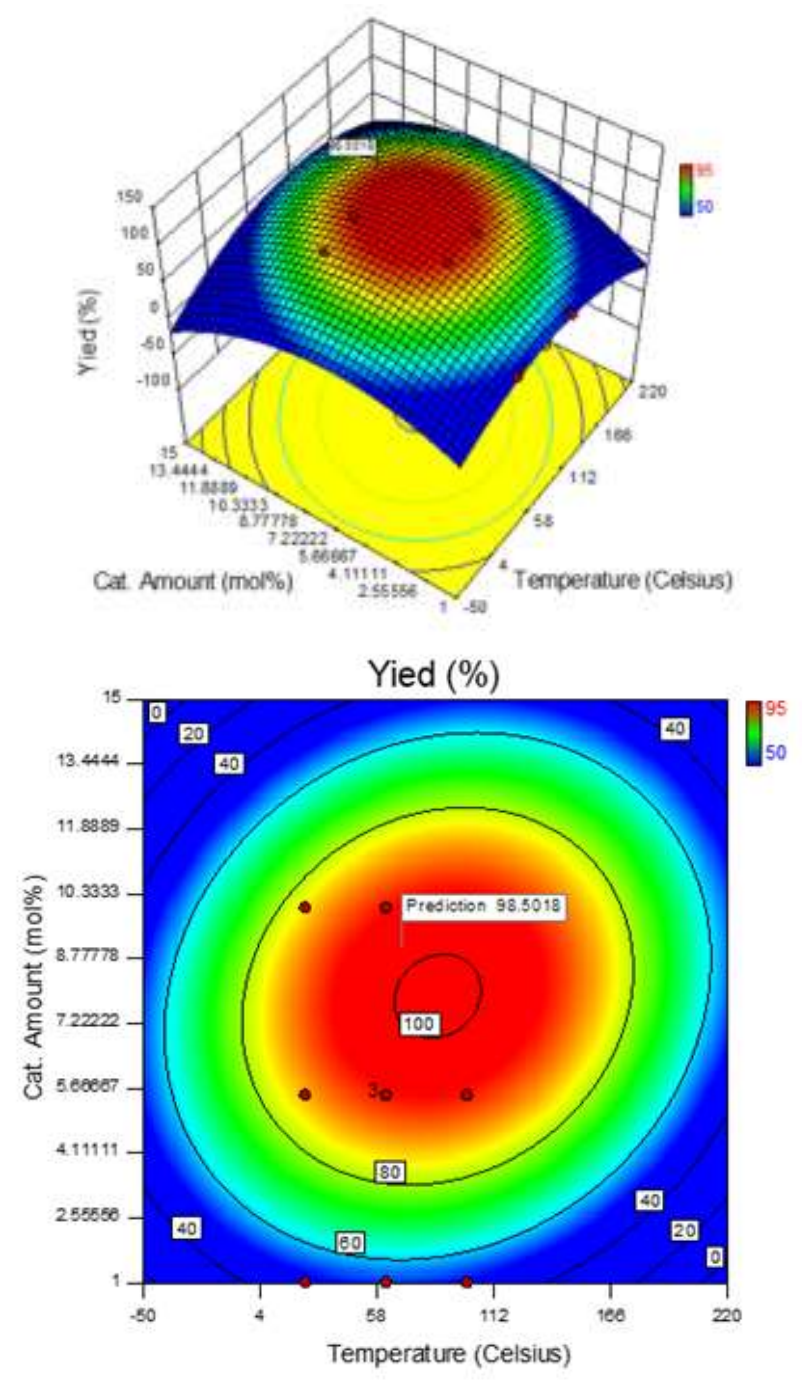

Figure 3. Three and two dimensional response surfaces for the effect of factors on yield of reaction

Further experiments, to compare the showed that reaction was more favoured efficiency of the solvent-free versus than the other conditions (such as $\mathrm{CHCl}_{3}$, solvent conditions, were conducted at the EtOAc, EtOH, $\mathrm{H}_{2} \mathrm{O}$, Acetone and THF). optimal point (Table 2) and results

Table 2. Effect of various solvents on the reaction of dimedone ( $2 \mathrm{mmol})$, 2-nitrobenzaldehyde (1 $\mathrm{mmol})$, and ammonium acetate $(1.2 \mathrm{mmol})$, in the persence of $\mathrm{Fe}_{3} \mathrm{O}_{4}(9 \mathrm{~mol} \%)$ -

\begin{tabular}{ccccc}
\hline Entry & Solvent & Temp. $\left({ }^{\circ} \mathbf{C}\right)$ & Time $($ min) & Yield $^{a}(\boldsymbol{\%})$ \\
\hline $\mathbf{1}$ & $\mathrm{CHCl}_{3}$ & Reflux & 20 & 85 \\
$\mathbf{2}$ & $\mathrm{EtOAc}$ & 70 & 25 & 85 \\
$\mathbf{3}$ & $\mathrm{EtOH}$ & 70 & 25 & 83 \\
$\mathbf{4}$ & $\mathrm{CH}_{2} \mathrm{Cl}_{2}$ & Reflux & 30 & 79 \\
$\mathbf{5}$ & $\mathrm{H}_{2} \mathrm{O}$ & 70 & 45 & 50 \\
$\mathbf{6}$ & $\mathrm{Acetone}$ & Reflux & 15 & 85 \\
$\mathbf{7}$ & $\mathrm{THF}$ & Reflux & 30 & 80 \\
$\mathbf{8}^{\boldsymbol{b}}$ & - & 70 & 4 & 95 \\
\hline
\end{tabular}

${ }^{\mathrm{a}}$ Isolated yield. ${ }^{\mathrm{b}}$ The reaction was proceeded in the absence of solvent.

To study the efficacy and the reacted with various aromatic aldehydes generality of the catalyst, dimedone was (aromatic as well as aliphatic containing 
electron-withdrawing groups, electrondonating groups and halogens) and ammonium acetate under the optimized reaction conditions (Scheme 2). As it is<smiles>CC1(C)CC(=O)c2c(nc3c(c2C(=O)c2ccccc2Cl)C(=O)CC(C)(C)C3)C1</smiles>

(1) $\mathrm{H}$

Time $(\min ): 4$ Yield (\%): 95 $\mathrm{mp}\left({ }^{\circ} \mathrm{C}\right): 221-227$ $[221-223]^{25}$<smiles>CC1(C)CC(=O)C2=C(C1)NC1=C(C(=O)CC(C)(C)C1)C2c1ccc(Br)cc1</smiles>

(5) $\mathrm{H}$

$$
\text { Time (min): } 5
$$
Yield (\%): 94 $\mathrm{mp}\left({ }^{\circ} \mathrm{C}\right): 241-243$ $[241-243]^{28}$<smiles></smiles>

(2) $\mathrm{H}$

Time $(\min ): 7$ Yield (\%): 89 $\mathrm{mp}\left({ }^{\circ} \mathrm{C}\right): 205-207$

$[212-214]^{26}$<smiles>CC1(C)CC(=O)C2=C(C1)NC1=C(C(=O)CC(C)(C)C1)C2c1ccccc1[N+](=O)[O-]</smiles>

(6) $\mathrm{H}$

$$
\begin{gathered}
\text { Time (min): } 4 \\
\text { Yield (\%): } 95 \\
\mathrm{mp}\left({ }^{\circ} \mathrm{C}\right): 271-274 \\
{[280-281]^{23}}
\end{gathered}
$$

shown in Scheme 2, all reactions proceeded efficiently to give the desired 1, 8-dioxo-decahydroacridines in high yields and in short reaction times.<smiles>CC1(C)CC(=O)C2=C(C1)NC1=C(C(=O)CC(C)(C)C1)C2c1cccc(Cl)c1</smiles>

(3) $\mathrm{H}$

Time (min): 5 Yield (\%): 90 $\mathrm{mp}\left({ }^{\circ} \mathrm{C}\right): 218-219$

$[214-216]^{27}$<smiles>CC1(C)CC(=O)C2=C(C1)NC1=C(C(=O)CC(C)(C)C1)C2c1ccc([N+](=O)[O-])cc1</smiles>

(7) $\mathrm{H}$

$$
\begin{aligned}
& \text { Time (min): } 10 \\
& \text { Yield (\%): } 89 \\
& \mathrm{mp}\left({ }^{\circ} \mathrm{C}\right): 314-316 \\
& {[>300]^{29}}
\end{aligned}
$$<smiles>CC1(C)CC(=O)C2=C(C1)NC1=C(C(=O)CC(C)(C)C1)C2c1cccc(Cl)c1Cl</smiles>

(4) $\mathrm{H}$

$$
\begin{gathered}
\text { Time }(\min ): 6 \\
\text { Yield }(\%): 95 \\
\mathrm{mp}\left({ }^{\circ} \mathrm{C}\right): 208-210 \\
{[212-215]^{18}}
\end{gathered}
$$<smiles>CC1(C)CC(=O)C2=C(C1)NC1=C(C(=O)CC(C)(C)C1)C2c1ccc2ccccc2c1</smiles>

(8) $\mathrm{H}$

Time (min): 4

Yield (\%): 95 $m p\left({ }^{\circ} \mathrm{C}\right): 213-215$ $[204]^{30}$<smiles>CC1(C)CC(=O)C(C2C3=C(CC(C)(C)CC3=O)NC3=C2C(=O)CC(C)(C)C3)C(=O)C1</smiles>

(9) $\mathrm{H}$

Time (min): 5 Yield (\%): 95 $\mathrm{mp}\left({ }^{\circ} \mathrm{C}\right): 268-270$ $[282-284]^{26}$<smiles>CC1(C)CC(=O)C2=C(C1)N(c1ccccc1)C1=C(C(=O)CC(C)(C)C1)C2c1ccccc1</smiles>

(10)

$$
\begin{aligned}
& \text { Time (min): } 10 \\
& \text { Yield (\%): } 90 \\
& \mathrm{mp}\left({ }^{\circ} \mathrm{C}\right): 242-243 \\
& {[254-256]^{31}}
\end{aligned}
$$

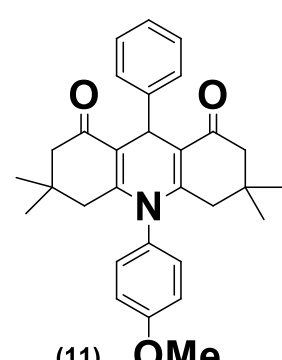

(11) OMe

Time (min): 8

Yield (\%): 89 $\mathrm{mp}\left({ }^{\circ} \mathrm{C}\right): 215-219$ $[215-219]^{23}$

Scheme 2. Scope of 1, 8-dioxo-decahydroacridines

To investigate the reusability of nano$\mathrm{Fe}_{3} \mathrm{O}_{4}$, after completion the reaction, the catalyst was collected by an external magnet and separated from product and remaining starting materials were tested again on the reaction of dimedone ( 2 mmol) with 2-nitrobenzaldehyde (1 mmol) and ammonium acetate (1.2 mmol) as a model reaction. As it is found in Figure 4, it is clarified that the catalyst could be reused for eleven times without significant changes in the yield of the product and reaction time. 


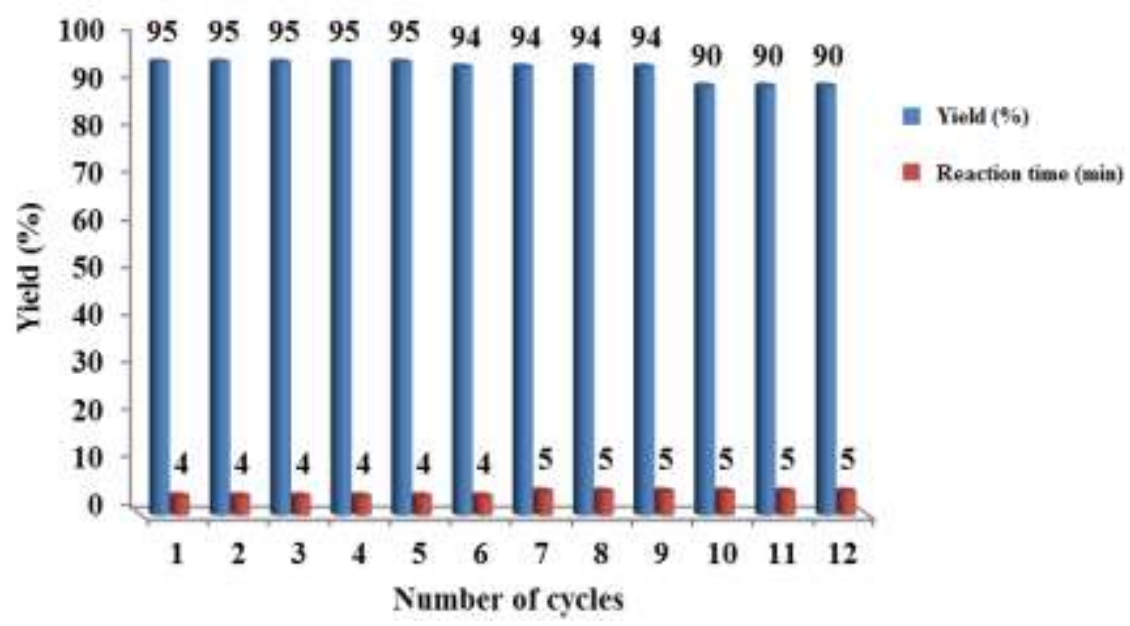

Figure 4. The reusability of the catalyst

\section{Conclusion}

In summary, we have reported nano$\mathrm{Fe}_{3} \mathrm{O}_{4}$ as a reusable, stable, general and heterogeneous catalyst for the preparation of 1, 8-dioxodecahydroacridines under solvent-free conditions. Also, the optimization of the reaction condition was investigated using the response surface method \{Central Composite Design (CCD)\} which was in a good accordance with empirical research.

\section{Acknowledgements}

We sincerely acknowledge the financial supports from Bu-Ali Sina University and Sayyed Jamaleddin Asadabadi University for supporting this work.

\section{References}

[1] a) A.R. Moosavi-Zare, M.A. Zolfigol, O. Khaledian, V. Khakyzadeh, M.D. Farahani, M.H. Beyzavi, H.G. Kruger, Chem. Engin. J., 2014, 248, 122127; b) A.R. Moosavi-Zare, H. Goudarziafshar, Z. Jalilian, Prog. Chem. Biochem. Res., 2019, 2, 59-63.

[2] A.R. Moosavi-Zare, M.A. Zolfigol, R. Salehi-Moratab, E. Noroozizadeh, $J$. Mol. Catal. A. Chem., 2016, 415, 144150.

[3] A.R. Moosavi-Zare, M.A. Zolfigol, S. Farahmand, A. Zare, A.R. Pourali, R.
Ayazi-Nasrabadi, Synlett., 2014, 25, 193-196.

[4] A. Khazaei, A.R. Moosavi-Zare, H. Afshar-Hezarkhani, V. Khakyzadeh, RSC Adv, 2014, 4, 32142-32147.

[5] A.R. Moosavi-Zare, M.A. Zolfigol, M. Daraei, Synlett, 2014, 25, 1173-1177. [6] S. Shylesh, V. Schunemann, W. R. Thiel, Angew. Chem. Int. Ed, 2010, 49, 3428-3459.

[7] Y. Zhu, L.P. Stubbs, F. Ho, R. Liu, C.P. Ship, J.A. Maguire, N.S. Hosmane, Chem. Cat. Chem., 2010, 2, 365-374.

[8] V. Polshettiwar, R.S. Varma, Org. Biomol. Chem, 2009, 7, 37-40.

[9] K.S. Lee, M.H. Woo, H.S. Kim, E. Y. Lee, I.S. Lee, Chem. Commun., 2009, 25, 3780-3782.

[10] V. Polshettiwar, Á. Molnár, Tetrahedron, 2007, 63, 6949-6976.

[11] M. Ma, Q. Zhang, D. Yin, J. Dou, H. Zhang, H. Xu, Catal Commun., 2012, 17, 168-172.

[12] L.M. Rossi, I.M. Nangoi, N.J.S. Costa, Inorg. Chem., 2009, 48, 46404642.

[13] M.A. Zolfigol, V. Khakyzadeh, A. R. Moosavi-Zare, A. Zare, P. ArghavaniHadi, Z. Mohammadi, M.H. Beyzavi, S. Afr. J. Chem., 2012, 65, 280-285.

[14] M.A. Zolfigol, A.R. Moosavi-Zare, P. Moosavi, V. Khakyzadeh, A. Zare, $C$. R. Chimie., 2013, 16, 962-966. 
[15] A. Davoodnia, A. Zare-Bidaki, H. Behmadi, Chin. J. Catal, 2012, 33, 17971801.

[16] K. Venkatesan, S.S. Pujari, K.V. Srinivasan, Synth. Commun., 2009, 39, 228-241.

[17] T.S. Jin, J.S. Zhang, T.T. Guo, A.Q. Wang, T.S. Li, Synthesis, 2004, 20012005.

[18] S. Rostamizadeh, A. Amirahmadi, N. Shadjou, A.M. Amani, J. Heterocyclic Chem., 2012, 49, 111-115. [19] B. Das, P. Thirupathi, I. Mahender, V.S. Reddy, Y.K. Rao, J. Mol. Catal. A. Chem., 2006, 247, 233-239.

[20] M. Dabiri, M. Baghbanzadeh, E. Arzroomchilar, Catal. Commun., 2008, 9, 939-942.

[21] W. Shen, L.M. Wang, H. Tian, J. Tang, J.J. Yu, J. Fluorine Chem., 2009, 130, 522-527.

[22] A. Davoodnia, H. Norouzi, N. Tavakoli-Hoseini, A. Zare-Bidaki, Synth. React. Inorg. Met. Org. Chem., 2015, 44, 70.

[23] A. Khazaei, A.R. Moosavi-Zare, Z. Mohammadi, V. Khakyzadeh, J. Afsar, J. Chin. Chem. Soc., 2016, 63, 165-170. [24] M.A. Ghasemzadeh, J. SafaeiGhomi, H. Molaei, C.R. Chimie., 2012, 15, 969-974.

[25] S. Tu, C .Miao, Y. Gao, F. Fang, Q. Zhuang, Y. Feng, D. Shi, Synlett., 2004, 2, 255-258.

[26] M.A. Zolfigol, N. Bahrami-Nejad, S. Baghery, J. Mol.Liq., 2016, 218, 558564.
[27] Zh. Obshch. Khim, Chinese J. Catal., 2016, 86, 1412-1418.

[28]. S. Balalaie, F.Chadegani, F.Darviche, H. R. Bijanzadeh, Chinese J Chem., 2009, 27, 1953-1956.

[29] G.H. Mohammadi Ziarani, A. Badiei, M. Hassanzadeh, S. Mousavi, Arab. Jour. Chem., 2011, 7, 335-339.

[30] A.K. Dutta, P. Gogoi, R. Borah, RSC Adv., 2014, 4, 41287-41291.

[31] A. Davoodnia, A. Khojastehnezhad, N. Tavakoli-Hoseini, Bull. Korean Chem. Soc., 2011, 32, 2243-2248.

[32] M.A. Zolfigol, A. Khazaei, A.R. Moosavi-Zare, A. Zare, V. Khakyzadeh, Appl. Catal. A: Gen., 2011, 400, 70-81. [33] A.R. Moosavi-Zare, M. A. Zolfigol, Z. Rezanejad, Can. J. Chem., 2016, 94, 626-630.

[34] A.R. Moosavi-Zare, M.A. Zolfigol, V. Khakyzadeh, C. Bottcher, M.H. Beyzavi, A. Zare, A. Hasaninejad, R. Luque, J. Mater. Chem. A., 2014, 2, 770777.

[35] A.R. Moosavi-Zare, M.A. Zolfigol, M. Zarei, A. Zare, V. Khakyzadeh, J. Mol. Liq., 2013, 186, 63-69.

[36] A. Khazaei, M.A. Zolfigol, A.R. Moosavi-Zare, F. Abi, A. Zare, H. Kaveh, V. Khakyzadeh, M. KazemRostami, A. Parhami, H. TorabiMonfared, Tetrahedron, 2013, 69, 212218.

[37] A.R. Moosavi-Zare, M.A. Zolfigol, M. Zarei, A. Zare, V. Khakyzadeh, A. Hasaninejad, Appl. Catal. A: Gen., 2013, 467, 61-68.

How to cite this manuscript: Ardeshir Khazaei, Ahmad Reza Moosavi-Zare, Hadis Afshar-Hezarkhani, Vahid Khakyzadeh. Programming of Fe-catalyzed cascade knoevenagel-michael-cyclocondensation reaction: create out pseudo acridine derivatives under solvent free conditions. Eurasian Chemical Communications, 2020, 2(1), 27-34. 